\title{
The Multiplex Phase Interlocker: a novel and robust molecular design synchronizing transcription and cell cycle oscillators
}

\author{
T.D.G.A. Mondeel ${ }^{1}$, C. Linke ${ }^{1}$, S. Tognetti ${ }^{2}$, W. Liebermeister ${ }^{3}$, M. Loog $^{4}$, \\ H.V. Westerhoff ${ }^{1}$, F. Posas ${ }^{2}$, M. Barberis ${ }^{1 *}$ \\ ${ }^{1}$ Swammerdam Institute for Life Sciences, University of Amsterdam, Amsterdam, The Netherlands \\ ${ }^{2}$ Departament de Ciències Experimentals i de la Salut, Universitat Pompeu Fabra, Barcelona, Spain \\ ${ }^{3}$ Institut für Biochemie, Charité - Universitätsmedizin Berlin, Berlin, Germany \\ ${ }^{4}$ Institute of Technology, University of Tartu, Tartu, Estonia \\ *e-mail:M.Barberis@uva.nl
}

Key words: network motifs, network dynamics, cell cycle, autonomous oscillations, timing, waves of cyclins, budding yeast, Multiplex Phase Interlocker

Motivation and Aim: The eukaryotic cell cycle is robustly designed, with molecules interacting and organized within definite network topologies. A transcriptional oscillator interlocks with waves of cyclin-dependent kinases (cyclin/Cdk) to guarantee execution of a timely cell cycle progression. Although details about transcription of cyclins, the regulatory subunits of these kinases, are available, a lack of understanding exists about network motifs responsible for the precise timing of cyclin/Cdk oscillations. Here we investigate the robustness of molecular designs interlocking the transcriptional and cyclin/Cdk oscillators in budding yeast. We have recently identified a transcriptional cascade that regulates the relative timing of waves of mitotic $(\mathrm{Clb})$ cyclin expression, which involves the Forkhead (Fkh) transcription factors (TF) [1]. Here we aim to unravel the network motif(s) responsible for timely cyclin/Cdk oscillations that interlock $\mathrm{Clb}$ waves through Fkh-mediated signaling.

Methods and Algorithms: An integrated computational and experimental framework is presented. A kinetic, ODE model of the cyclin/Cdk network is simulated under a quasi-steady state assumption, and fitted to in vivo quantitative, time course data of $\mathrm{Clb}$ dynamics. Robustness analyses are then performed by testing 1024 possible network motifs for their ability (i) to fit $\mathrm{Clb}$ oscillations and (ii) to generate sustained oscillations in the form of limit cycles, on which sensitivity analysis is conducted.

Results: A novel regulatory motif, coined as Multiplex Phase Interlocker, is unraveled, that timely synchronize $\mathrm{Clb}$ oscillations. This motif uniquely describes a molecular timer (TF) that relies on separate inputs ( $\mathrm{Clb} / \mathrm{Cdk}$ ) converging on a common target (TF itself). Within the motif, a progressive TF activation may be realized by multiple $\mathrm{Clb} / \mathrm{Cdk}$. Experimental validation supports computational analyses, with the Clb/Cdk-Fkh axis being pivotal for timely transcriptional dynamics.

Conclusion: Altogether, our integrative approach pinpoints how robustness of cell cycle control is realized by revealing a novel and conserved principle of design that ensures a timely interlock of transcriptional and cyclin/Cdk oscillations.

Acknowledgements: Supported by the SILS Starting Grant of the University of Amsterdam, UvA and by the UvA-Systems Biology Research Priority Area Grant.

\section{References}

1. Linke C., Chasapi A., González-Novo A., Al Sawad I., Tognetti S., Klipp E., Loog M., Krobitsch S., Posas F., Xenarios I., Barberis M. (2017) A Clb/Cdk1-mediated regulation of Fkh2 synchronizes CLB expression in the budding yeast cell cycle. Nature NPJ Systems Biology and Applications. 3:7. 